\title{
Educación para jóvenes y adultos: visibilizando diversas trayectorias educativas
}

\author{
Tabata Contreras-Villalobos ${ }^{1}$ y Enrique Baleriola ${ }^{2}$ \\ Pontificia Universidad Católica de Valparaíso-Chile y Universitat Oberta de \\ Catalunya-España
}

\begin{abstract}
Los desafíos por una educación de calidad son complejos dada las cifras de deserción escolar. En Chile, esta población es atendida por la modalidad Educativa para Jóvenes y Adultos, donde no existe suficiente información sobre estas trayectorias educativas. Esta investigación cuantitativa, tuvo como objetivo caracterizar a estos estudiantes, indagando sobre cómo abordar la deserción. Los resultados caracterizan a una población mayoritariamente masculina, menores de veinticinco años, con alta inasistencia y bajas calificaciones. Desde el MRL, asistencia, género y nivel de enseñanza son los factores que más explican el rendimiento escolar y la aprobación, determinando la continuidad de la trayectoria escolar. Para brindar oportunidades para el aprendizaje a lo largo de la vida, se sugieren estrategias de intervención con un enfoque sistémico.
\end{abstract}

Palabras clave: educación para jóvenes y adultos, trayectoria educativa, deserción escolar.

\section{Education for youth and adults: Making visible diverse educational trajectories}

The challenges for quality education are complex given the high dropout rates. In Chile, this population is managed by the Youth and Adult Education modality, which there is not enough information on these educational trajectories. The aim of this quantitative research was to characterize these students, inquiring about how to tackle school dropout. The results show a mostly male population, under twenty-five years, with low attendance and low marks. From the MLR analysis, attendance, gender and level of education are the factors that most explain school performance, determining the continuity of the school trajectory. In order to provide new opportunities for lifelong learning, it is suggested to focus on intervention strategies with a systemic approach.

Keywords: education for young people and adults, educational trajectory, school dropout.

\section{Educação para jovens e adultos: visitando diversas trajetórias educacionais}

Os desafios para uma educação de qualidade são complexos, dadas as taxas de abandono escolar. No Chile, esta população é servida pela modalidade de Educação de Jovens e Adultos, onde não existe informação suficiente sobre estas trajectórias educacionais. O objectivo desta

1 Magister en Educación. Estudiante de Doctorado en la Pontificia Universidad Autónoma de Valparaíso, Chile. Dirección postal: Avenida El Bosque, 1290. Viña del Mar, región de Valparaíso, Chile. Contacto: Tabata.contreras@pucv.cl. https://orcid.org/0000-0002-6579-9269

2 Doctor en Psicología Social. Profesor lector en la Universidad Oberta de Catalunya, Espańa. Dirección postal: Rambla del Poblenou, 150. Barcelona, España. Contacto: ebaleriola@uoc. edu. https://orcid.org/0000-0003-2899-6316 
investigação quantitativa era caracterizar estes estudantes, inquirindo sobre a forma de lidar com o abandono escolar. Os resultados caracterizam uma população maioritariamente masculina, com menos de vinte e cinco anos de idade, com altas não presenças e baixas notas. A partir do MRL, frequência, sexo e nível de educação são os factores que mais explicam o desempenho escolar, determinando a continuidade da carreira escolar. Sugere-se que se centre nas estratégias de intervenção com uma abordagem sistémica, proporcionando novas oportunidades de aprendizagem ao longo da vida.

Palavras-chave: educação de jovens e adultos, caminho educativo, deserto escolar.

\section{Éducation des jeunes et des adultes: visite de trajectoires éducatives diverses}

Les défis à relever pour une éducation de qualité sont complexes compte tenu des taux d'abandon scolaire. Au Chili, cette population est desservie par la modalité d'éducation des jeunes et des adultes, où il n'y a pas assez d'informations sur ces trajectoires éducatives. L'objectif de cette recherche quantitative était de caractériser ces élèves, en s'interrogeant sur la manière de lutter contre le décrochage scolaire. Les résultats caractérisent une population majoritairement masculine, âgée de moins de vingt-cinq ans, avec un taux d'absentéisme élevé et des notes faibles. D'après le MRL, l'assiduité, le sexe et le niveau d'éducation sont les facteurs qui expliquent le plus la performance scolaire, déterminant la continuité du parcours scolaire. Il est suggéré de se concentrer sur les stratégies d'intervention avec une approche systémique, en offrant de nouvelles possibilités d'apprentissage tout au long de la vie.

Mots-clés: éducation des jeunes et des adultes, parcours éducatif, désertion scolaire. 
Según la Organización de las Naciones Unidas (ONU), la educación forma la base para la formación de las personas, siendo un derecho que debe ser cumplido desde sus niveles iniciales hasta la secundaria, permitiendo un desarrollo sostenible y una mejora en la calidad de vida (ONU, 2015a). Consecuentemente, el concepto de calidad de educación se ubica en el centro de discusiones internacionales y forma parte de diversos compromisos asumidos por los gobiernos, como uno de los objetivos principales de la agenda de 2030 (Unesco, 2017; CPCE, 2016). Parte de una educación de calidad, es que esta tenga un carácter inclusivo y equitativo, ya que elimina barreras y entrega a la población herramientas necesarias para desarrollar soluciones innovadoras a los problemas del mundo actual (Echeita y Verdugo, 2004; Ainscow, 2017).

Pero a pesar de la centralidad de la educación en la sociedad encontramos que, en la actualidad, más de 265 millones de personas en el mundo no acuden a clase. Si bien, se han producido importantes avances en la tasa de escolarización, mejora en cobertura, acceso a todos los niveles educativos, e incluso en los niveles de alfabetización; tal como expresa la Organización para la Cooperación y el Desarrollo Económico (OCDE, 2016) aún queda mucho por avanzar para que estas personas tengan las competencias necesarias, culminen su educación obligatoria y tengan los conocimientos teóricos y prácticos para su desarrollo sostenible (ONU, 2015b).

En el caso particular de Latinoamérica, el no terminar la educación obligatoria arrastra costos enormes como menor cohesión social, menor posibilidad de movilidad social y menor participación en actividades cívicas y políticas (OCDE, 2015). Estos factores aumentan las brechas de desigualdad y vulnerabilidad social, llevando a que en múltiples cumbres y reuniones de autoridades de la región se haya señalado la importancia de la educación y, en particular, de la educación de 
personas jóvenes y adultas para combatir esta problemática (Campero, 2018). Si bien se han desarrollado iniciativas y avances en educación para reducir estas brechas, el ritmo de este progreso es muy lento, afectando a los 35 millones de adultos que todavía carecen de competencias básicas en lectura y escritura, o a los 88 millones de mujeres y hombres que no han completado la educación primaria; siendo muchos más quienes abandonaron y no han completado la educación secundaria (Unesco, 2017). Estas tensiones son las que bordean uno de los principales problemas educativos: la deserción escolar (Cortés et al., 2019).

En el caso de Chile, la deserción escolar a nivel nacional ha bajado ininterrumpidamente entre los ańos 2012 (3.7\%) y 2018 (2.1\%). Aunque estas últimas cifras parecen pequeñas, encontramos que son más de 30.000 personas las que salieron del sistema educativo regular. En cambio, si atendemos a la población joven y adulta, el porcentaje asciende hasta un $32.5 \%$ de estudiantes que desertan y no terminan su año escolar. Estos forman parte de la modalidad Educativa Para Jóvenes y Adultos (EPJA) (Mineduc, 2018).

Esta modalidad educativa busca revertir las consecuencias que arrastra el no terminar la educación obligatoria. Así, la educación para jóvenes y adultos acoge a las personas que han desertado de la educación regular, asegurando su derecho a la educación y procurando que puedan terminar su trayectoria educativa. Sin duda, la incorporación del estudiante a esta modalidad repercute en sus vidas, pues el reingreso a la educación formal en la modalidad EPJA y su culminación, promueve el acceso a instituciones sociales, culturales, políticas y económicas mediante la acreditación de niveles de aprobación y la pertenencia a una comunidad (Opazo, 2017; Morales, 2011).

De esta forma, la modalidad educativa para jóvenes y adultos supone un espacio público de transformación y nueva oportunidad para aquellas personas que han desertado de la educación obligatoria. En Chile, la misión de EPJA es asegurar el acceso a un sistema educativo de calidad, adecuada a los intereses, características y necesidades de sus estudiantes, promoviendo la formación integral y permanente de las personas (Mineduc, 2017). Una característica importante de 
esta modalidad es la posibilidad de inscripción para todas las personas interesadas, sin importar edad, nacionalidad, situación migratoria o situación penal o de privación de libertad.

En las investigaciones desarrolladas en Chile en los últimos años, la información respecto a los Centros Educativos para Jóvenes y Adultos se centra principalmente en definir perfiles estudiantiles y dar cuenta de la reescolarización (Díaz et al., 2016; Espinoza et al., 2014). Si bien estas han sido un aporte para conocer esta realidad educativa, parten de una perspectiva local e individualizadora, centrada exclusivamente en el estudiante y obviando el papel de toda la comunidad educativa, de la gestión y de las trayectorias con las que trabajan. No obstante, nos permiten comprender algunas características: se ha identificado una alta heterogeneidad en la población que asiste a esta modalidad de educación. En ese sentido, se trata de una población en la que se mezclan personas con diferentes grados de vulnerabilidad, ya sea por su edad, género, nacionalidad, etc., con el fin de integrarse socialmente (Dussaillant, 2017). La media en edad ha bajado en los estudiantes de estos centros (Muñoz y Acuña, 2013) y no se advierte una reestructuración acorde a este desafío: estrategias pedagógicas, mayor dotación de recursos humanos y materiales disponibles (Castillo et al., 2013).

La EPJA se tiende a concebir como un sub-sistema cuyo foco principal debe ser la alfabetización, la educación compensatoria de las personas jóvenes y adultas que en algún momento de la vida abandonaron la escuela. Sin embargo, esa visión no responde a los desafíos actuales de la educación en el siglo XXI, ni con el enfoque de la educación a lo largo de la vida, ni con la educación popular tan característica de la región latinoamericana (Infante y Letelier, 2013). Es ante este panorama surge el presente estudio, cuyo objetivo consiste en ofrecer una caracterización de la población EPJA, describiendo sus procesos de aprendizaje y aproximándonos a sus trayectorias educativas. De esta forma, podremos arrojar luz de cómo abordar y gestionar procesos de transformación y mejoras educativas en una población de la que aún existen pocos datos y estudios al respecto. 
Ya para el año 2018, la matrícula en la modalidad EPJA ascendía a más de 140.000 estudiantes lo que representa aproximadamente un $4 \%$ del total de estudiantes de todo el sistema educativo (desde educación parvularia a superior), alojada en un $10 \%$ de los establecimientos educativos de Chile (Mineduc, 2019). Si bien esta matrícula se ha mantenido estable en los últimos cinco años, ha aumentado la cantidad de estudiantes que logran culminar la educación para jóvenes y adultos, casi doblando su número entre 2007 (31.133 estudiantes) a 2017 (59.346) (Mineduc, 2018). Estas cifras siguen representando un desafío del cual la modalidad EPJA debe afrontar.

Algunas de las causas de estas alarmantes cifras y la escasez de trabajos al respecto la encontramos en que, en Chile, la educación pública es la gran perjudicada por el modelo mercantil neoliberal que hoy impera, producto de diferentes mecanismos performativos de privatización, estandarización y rendición de cuentas (Carrasco, 2013), y de lógicas de nuevo management público que generan una alta competencia para las escuelas, mediante estándares e incentivos económicos ligados a resultados (Fardella, 2013; Soto et al., 2016). Esta lógica neoliberal apenas se lleva a cabo en EPJA, pero tampoco es tratada de forma diferenciada para contribuir a su gestión e inclusión. Y por tanto, no reciben una subvención escolar preferencial acorde al contexto vulnerable de esta modalidad educativa, como sí sucede en las escuelas públicas regulares (Mineduc, 2016). Por el contrario, existe una brecha en la educación para jóvenes y adultos dada la diversidad que se da en ella (de edad, nacionalidad, raza, niveles de enseńanza, etc.), constituyendo una desigualdad en el trato y en el apoyo proporcionado (DVV International, 2018) y concibiendo que los problemas de deserción son de origen individual, a la que se le atribuyen resultados y mediciones estandarizados (Terigi, 2007). Esto sitúa a EPJA en un limbo fuera de reformas y decretos sobre si debe rendir cuentas o no, de apoyo directo a las escuelas, o incluso sobre su privatización. Si la educación pública en Chile ha venido en desmedro, la educación para jóvenes y adultos ha sido la gran olvidada y precarizada (Guerrero, 2017), lo que finalmente solo termina por perjudicar más aún a los y las estudiantes de esta modalidad. 
Y esto, pese a que las diferentes instituciones nacionales e internacionales mantienen la promesa de asegurar la educación inclusiva, equitativa y de calidad, donde se promuevan oportunidades de aprendizaje a lo largo de la vida para todos y todas (Unesco, 2019).

\section{Trayectoria educativa. Una aproximación a la realidad de EPJA}

Según la Unesco $(2015,2017)$, EPJA se debe desarrollar bajo un marco de inclusión que responda a la diversidad propia de esta modalidad. Este enfoque se relaciona con el acceso, la permanencia, la participación de los estudiantes, pero sobre todo con sus logros, permitiendo una transformación en el entendimiento de la desescolarización, desde concebirse como un fracaso escolar individual - la no aprobación del curso educativo que está la persona ya sea por rezago, repetición o desafiliación-, hacia un entendimiento de esta problemática desde los factores socioeducativos que llevan a la deserción escolar (CPCE, 2016; Aristimuño, 2015). De esta forma, se avanza hacia una comprensión y gestión de cómo retener a los estudiantes para que estos egresen finalmente de la educación obligatoria atendiendo a la pluralidad de trayectorias y características de cada estudiante de EPJA. Bajo esta óptica, no son los estudiantes los que fracasan, sino que es el sistema educativo el que no logra dar respuesta a las particulares necesidades de esta población, reforzando la perspectiva inclusiva y sistémica promovida por la Unesco, que busca poner foco en la retención escolar y repercutir finalmente en las trayectorias educativas (Mayer y Cerezo, 2016; Espinoza et al., 2019).

Las trayectorias educativas, por tanto, son los recorridos efectivos que realizan los sujetos en el sistema escolar, comparados con la expectativa que de ellos espera el diseño sociopolítico (Terigi, 2007). Este diseño responde a un ideal de recorridos de progresión lineal marcados por tiempos estandarizados, condicionando niveles con edades, sin que se repita ni se abandone ese grado. Es por esto que se llaman teóricas, pero no siempre se consiguen, surgiendo el concepto de trayectorias escolares reales, donde encontramos recorridos que no siguen ese cauce, reconociendo que muchos de los estudiantes llevan a cabo su proceso 
de modo heterogéneo, variable y contingente (Terigi, 2012). Son estas trayectorias reales las que encontramos más explícita y frecuentemente en la modalidad para jóvenes y adultos, donde se introducen las opciones de: no ingresar al sistema educativo, hacerlo tardíamente, abandonar temporalmente, repetir algún nivel (más de una vez), o incluso permanecer sin lograr los aprendizajes esperados. Esto se puede deber a condiciones personales, pero también a condiciones institucionales (Terigi, 2014), por tanto, se debe poner atención tanto a estas trayectorias como a los procesos de deserción que las acompañan, a los contextos, y a una lectura sistémica para así contribuir a la consecución de una educación inclusiva y de calidad.

Actualmente, no existe información disponible que permita realizar el seguimiento de trayectorias escolares en la población chilena de EPJA. Pero sí existe información de asistencia, matrícula, logro, repetición y sobreedad desde las instituciones oficiales; lo que permite acercarnos a los y las estudiantes, a sus recorridos y a sus experiencias; en su relación con el sistema escolar, permanencia y egreso. Así, dada la escasa información disponible sobre las trayectorias de los estudiantes que llevan a la deserción escolar en EPJA, es que esta investigación tiene por objetivo caracterizar las trayectorias educativas de esta población y su relación con los procesos de deserción escolar que experimentan. Con esto, se pretende arrojar luz sobre cómo abordar y gestionar procesos de transformación educativa que mejoren la calidad en EPJA. Para ello, hemos planteado una investigación cuantitativa que tiene como hipótesis: 1) existe una relación positiva según género y edad con la tasa de aprobación final, privilegiando a mujeres y personas mayores; 2) existe una relación positiva entre tasa de asistencia y promedio de notas, con la tasa de aprobación final y 3), se espera encontrar diferencias significativas en cuanto al nivel educativo y la edad en los resultados académicos que obtienen. 


\section{Método}

\section{Participantes}

Para este estudio, participaron (n) 29.035 estudiantes que asistieron a la modalidad EPJA en el año 2018, correspondiente a los registros e información disponible por las bases de datos públicas de Chile. De estos, 17.128 (59\%) eran hombres y 11.907 (41\%) mujeres entre los 13 y 87 años $(M=25.4, D E=10.6)$, pertenecientes a los niveles de educación básica (5.143), educación secundaria humanista científico (20.814) y educación secundaria técnico profesional (3078) de las diversas regiones del país. Si bien en los registros se habla de un número mayor en la matrícula de estudiantes en la modalidad educativa, este número disminuye al realizarse una limpieza de la información, por tanto, se pudo acceder solo a las bases de datos con información completa proporcionada por los establecimientos educacionales a los organismos centrales.

\section{Instrumento y variables}

Se acudió a bases de datos públicas oficiales provenientes del Ministerio de Educación, información que se encuentra disponible en la sección de Datos abiertos del centro de estudios del Mineduc. En esta plataforma, se solicitaron dos bases de datos: a) número de matrícula de estudiantes para el año 2018, b) las tasas de aprobación y suspensos del año 2018.

Para este análisis, sobre la base del objetivo propuesto se utilizaron las variables demográficas sobre 1) Niveles de enseñanza a los que pertenecen los estudiantes, divididos en un nivel Básico o primaria, y en secundaria se divide entre las especialidades de: Educación Media Humanístico científico y Educación Media Técnico profesional, 2) Edad de las personas comprendida como años cumplidos al 30 de junio en el 2018; y 3) Sexo de los estudiantes. En cuanto al aproximamiento a las trayectorias educativas de los estudiantes, se consideró 1) asistencia, comprendida como porcentaje de asistencia anual en 
la escuela; 2) notas de los estudiantes, entendido como el promedio general anual de cada estudiante, medido en escala de 1 a 7 ; y 3) tasa de aprobación, comprendida como el estado final al cierre del año de cada estudiante, que podía ser: promovido o aprobado (que pasa de grado), reprobado (no logra pasar de grado), traslado (la persona se retira del establecimiento educacional, pero registra matrícula en otro lugar) o retirado (la persona se retira del establecimiento educacional y no hay más registros de matrícula).

\section{Procedimiento}

Luego de la obtención de la base de datos, se identificaron las variables desde el libro de códigos proporcionado. Se continuó con una fusión de estas bases a través del RBD (número identificador de las escuelas), donde se realizó una limpieza de la información. Además, se recodificaron ciertas variables, agrupando los niveles educativos por escuelas para facilitar los análisis. Cabe destacar, que las bases no vienen con datos perdidos tal como se mencionó anteriormente.

\section{Análisis de datos}

Los análisis se realizaron con el programa SPSS versión 25, este software facilitaba el trabajo a partir del formato de BBDD proporcionada. Para este caso, en primer lugar, se realizó un análisis descriptivo, utilizando un análisis de frecuencia para caracterizar en mayor detalle a estos estudiantes y sus trayectorias educativas. Posteriormente, se realizó un análisis estadístico inferencial, para conocer si es que existe relación entre las variables, sobre todo entre notas, porcentaje de asistencia, edad, sexo y tasa de aprobación, para comprobar las hipótesis planteadas. Para esto es que se acude a realizar tablas de contingencia con un análisis de Chi cuadrado para aquellas variables nominales, y análisis correlacionales para las variables continuas. Finalmente, a través del Modelo de Regresión Lineal (MRL) se realiza un análisis para comprender la tasa de aprobación de los estudiantes, considerando las variables sobre porcentaje de asistencia, notas, edad y género. 


\section{Consideraciones éticas}

Para esta investigación, se solicitó la información por ley 20.285 de transparencia del Gobierno de Chile (2015), donde se aseguró que los datos proporcionados fueron utilizados solo con fines científicos, resguardando integridad y anonimato de las escuelas, sus estudiante y resultados. Posteriormente, para conseguir los objetivos planteados, se acudió a la información que proporciona el Centro de Estudios del Mineduc, portal de datos abiertos de la educación chilena con información detallada del sector educativo, de libre acceso a bases de datos.

\section{Resultados}

En primer lugar, describiremos algunos puntos esenciales los y las estudiantes de esta modalidad educativa, acercándonos a sus trayectorias atendiendo a 1) su caracterización según tasa de aprobación, 2) las relaciones entre nivel de enseñanza, notas y edad, 3) la relación entre notas, asistencia y edad, 4) la comparación entre nivel de enseñanza y porcentaje de asistencia, y 5) la comparación entre sexo y porcentaje de asistencia. Posteriormente, ofreceremos los estadísticos apropiados para el contraste de las hipótesis de modelos explicativos.

En este sentido es que en la tabla 1 se pueden observar las características esenciales de este grupo, clasificados por la tasa final de aprobación. Podemos identificar que mayoritariamente las personas que aprueban son mujeres, con una alta asistencia (más del 50\%). En el caso de las personas que reprueban, mayoritariamente son hombres, entre 19 a 25 años de edad, que están cursando una educación secundaria técnico profesional, donde sus notas son menor a 4 en una escala de 1 a $7(M=3.1, D E=2.6)$, y que su porcentaje de asistencia es muy bajo, menor al 25\% $(M=52.1, D E=43.3)$. Estas cifras son más severas para aquel grupo que se retira de la modalidad, es decir, vuelve a desertar del sistema educativo. Cabe destacar que las personas de este grupo son la segunda mayoría, es decir, luego del grupo de aprobados donde encontramos que un 54\% de las mujeres y un $46 \%$ de los 
hombres logran pasar de grado al finalizar el año, un 27\% de mujeres y $30 \%$ de los hombres se retiran de la modalidad educativa para jóvenes y adultos en el año 2018 .

\section{Tabla 1}

Caracterización de la población según tasa de aprobación (\%)

\begin{tabular}{|c|c|c|c|c|c|}
\hline & & Promovido & Reprobado & Traslado & Retirado \\
\hline \multirow[t]{2}{*}{ Sexo } & Masculino & 45.9 & 15.1 & 9.4 & 29.5 \\
\hline & Femenino & 54 & 10.9 & 8.3 & 26.8 \\
\hline \multirow[t]{4}{*}{ Edad } & 13 a 18 & 51 & 15.3 & 11.9 & 21.8 \\
\hline & 19 a 25 & 43.8 & 13.7 & 9.2 & 33.4 \\
\hline & 26 a 50 & 52.4 & 11.3 & 6.3 & 30 \\
\hline & $>50$ & 58.8 & 11.1 & 3.8 & 26.4 \\
\hline \multirow[t]{3}{*}{ Nivel de enseńanza } & Básica & 50.1 & 15.1 & 7.7 & 27. \\
\hline & Media HC & 49.4 & 13.1 & 9.1 & 28.3 \\
\hline & Media TP & 46.7 & 11.9 & 9.9 & 31.5 \\
\hline \multirow[t]{4}{*}{ Notas } & 0 a 3.9 & 0 & 21.3 & 18.9 & 59.8 \\
\hline & 4 a 4.9 & 77.3 & 22.7 & 0 & 0 \\
\hline & 5 a 5.9 & 99.2 & 0.8 & 0 & 0 \\
\hline & 6 a 7 & 100 & 0 & 0 & 0 \\
\hline \multirow[t]{4}{*}{ Asistencia } & 0 a $25 \%$ & 0.2 & 4.7 & 22.8 & 72.2 \\
\hline & 26 a $50 \%$ & 21.3 & 78.7 & 0 & 0 \\
\hline & 51 a $75 \%$ & 61.9 & 38 & 0 & 0 \\
\hline & 76 a 100 & 88.4 & 11.6 & 0 & 0 \\
\hline
\end{tabular}

En cuanto a las diferencias respecto al nivel de enseñanza, se puede observar en la Tabla 2 que los y las estudiantes de la modalidad 
educativa para jóvenes y adultos pertenecientes a la enseñanza básica o primaria, son quienes presenta mayor diferenciación con los otros niveles educativos. Por ejemplo, en cuanto a edad $(M=31, D E=14)$ son el nivel educativo que en promedio es mayor en ańos y presentan mayor asistencia $(M=56, D E=45)$. Por otra parte, llama la atención que aquellas personas que están cursando una educación media técnica profesional presentan la menor asistencia $(M=48, D E=43)$ y menor promedio de notas $(M=3.1, D E=2.7)$. A pesar de esto, se puede apreciar transversalmente que los resultados académicos son bajos, con una desviación bastante alta, demostrando una amplia diversidad al respecto.

\section{Tabla 2}

Asistencia, promedio de notas y edad, según nivel de enseñanza

\begin{tabular}{lcccccc}
\hline Nivel de enseñanza & \multicolumn{2}{c}{ \% Asistencia } & \multicolumn{2}{c}{ Promedio Notas } & \multicolumn{2}{c}{ Edad } \\
\cline { 2 - 7 } & $M$ & $D E$ & $M$ & $D E$ & $M$ & $D E$ \\
\hline Básica & 56 & 45 & 3.2 & 2.5 & 31 & 14 \\
Media HC & 52 & 43 & 3.2 & 2.6 & 24 & 9 \\
Media TP & 48 & 43 & 3.1 & 2.7 & 26 & 9 \\
\hline
\end{tabular}

En cuanto a la asociación de estas variables, la tabla 3 indica que existe una correlación positiva alta $(r=.95, p<.01)$ entre Notas y Asistencia, es decir a mayor asistencia, más alta es la nota y viceversa. Este también es el caso entre edad y notas $(r=.07, p<.01)$, mientras mayor es la persona, más alta su calificación. Por último, se puede observar una correlación entre Asistencia y Edad $(r=.08, p<.01)$, donde a mayor edad encontramos más asistencia. Por tanto, a estas variables de notas, asistencia y edad debemos poner atención ante la gestión de las trayectorias educativas, sobre todo cuando las personas que son parte de la modalidad educativa ahora son mayoritariamente jóvenes, con baja tasa de asistencia y bajo promedio de notas, las que podrían estar en riesgo de desertar nuevamente. 


\section{Tabla 3}

Correlación entre notas, asistencia y edad

\begin{tabular}{lccc}
\hline & 1 & 2 & 3 \\
\hline 1. Notas & - & $.945^{* *}$ & $.072^{* *}$ \\
2. Asistencia & & - & $.082^{* *}$ \\
3. Edad & & & - \\
\hline
\end{tabular}

Al buscar conocer las diferencias que se tienen inter e intra grupos en la modalidad de EPJA, es que se acudió a la comparación de grupos, dónde tal como muestra la tabla 4, se encontraron diferencias entre el porcentaje de asistencia y el nivel de enseñanza al que asisten estos estudiantes, con un $F(2 ; 20.032)=41.052, p<.01$, indicando que existen diferencias significativas entre los distintos niveles, donde efectivamente son los estudiantes de educación media técnico profesional quienes tienen peor asistencia en comparación a las otras modalidades.

\section{Tabla 4}

Comparación de media entre nivel de enseñanza y porcentaje de asistencia en EPJA

\begin{tabular}{llccccc}
\hline \multirow{2}{*}{$\begin{array}{c}\text { Nivel de enseñanza } \\
\text { agrupados }\end{array}$} & $\begin{array}{c}\text { Diferencia de } \\
\text { medias (I-J) }\end{array}$ & $D E$ & $p$ & & \multicolumn{2}{c}{ IC al 95\% } \\
\cline { 6 - 7 } Básica & Media HC & $4.61^{*}$ & .67 & .00 & 3.03 & Límite inferior \\
& Media TP & $8.58^{*}$ & .98 & .00 & 6.27 & 10.88 \\
Media & Básica & $-4.61^{*}$ & .67 & .00 & -6.19 & -3.03 \\
HC & Media TP & $3.97^{*}$ & .83 & .00 & 2.01 & 5.92 \\
Media & Básica & $-8.58^{*}$ & .98 & .00 & -10.88 & -6.27 \\
TP & Media HC & $-3.97^{*}$ & .83 & .00 & -5.92 & -2.01 \\
\hline
\end{tabular}

En el caso de la comparación por sexo y la tasa de asistencia, la tabla 5 muestra que las diferencias son significativas, con un $\mathrm{F}(1 ; 29.033)$ $=17.198 ; \mathrm{p}<.01$, siendo las mujeres quienes asisten porcentualmente 
más durante el año escolar, a pesar de que mayoritariamente son hombres los matriculados en la modalidad educativa para jóvenes y adultos.

\section{Tabla 5}

Comparación de media entre sexo y porcentaje de asistencia en EPJA

\begin{tabular}{lccccc}
\hline & Suma de cuadrados & gl & Media cuadrática & $F$ & $p$. \\
\hline Entre grupos & 32154.506 & 1 & 32154.51 & 17.20 & .000 \\
Dentro de grupos & 54281809.379 & 29033 & 1869.66 & & \\
Total & 54313963.885 & 29034 & & & \\
\hline
\end{tabular}

En cuanto a la comparación de media entre promedio general y porcentaje de asistencia según edad, se indica que existen diferencias entre grupos, donde la traza de Pillai obtuvo $F_{(6 ; 58044)}=57.540, p<$ .001 . Al revisar en detalle estas diferencias, es que se expresa en la tabla 6 que tanto en notas $\left(F_{(3 ; 29021)}=88.016, p<.001\right)$ como asistencia $\left(F_{(3 ; 58044)}=107.837, p<.001\right)$ es significativa. En la prueba de post hoc, sobre el promedio general de notas, también se observan que las diferencias son significativas. Son aquellas personas que tienen 19 a 25 años las que presentan diferencias significativas de forma negativa con todos los otros grupos, es decir presentan los promedios de notas más bajos. Mientras de manera opuesta, encontramos que son las personas mayores de 50 años las que tienen diferencias significativas con todos los grupos, pero en este caso, las diferencias son positivas.

\section{Tabla 6}

Manova para promedio general y asistencia de notas, por edad de los estudiantes EPJA

\begin{tabular}{lcccccccc}
\hline Variable & $\begin{array}{c}\text { Tipo III de suma } \\
\text { de cuadrados }\end{array}$ & $\mathrm{gl}$ & $\begin{array}{c}\text { Media } \\
\text { cuadrática }\end{array}$ & $F$ & $p$. & $\begin{array}{c}\text { Eta parcial } \\
\text { al cuadrado }\end{array}$ & $\begin{array}{c}\text { Parámetro sin } \\
\text { centralidad }\end{array}$ & $\begin{array}{c}\text { Potencia } \\
\text { observada }\end{array}$ \\
\hline Notas & 1804.28 & 3 & 601.43 & 88.02 & 0 & .01 & 264.05 & 1 \\
$\begin{array}{l}\text { Porcentaje } \\
\text { asistencia }\end{array}$ & 598588.58 & 3 & 199529.53 & 107.84 & 0 & .01 & 323.51 & 1 \\
\hline
\end{tabular}


Sobre la base de los datos obtenidos, es que se plantea un modelo explicativo bajo el MRL correspondiente a la tabla 7 para la variable dependiente de asistencia, según género, edad y nivel de enseñanza, el cual explica un $0.9 \%$ de la varianza $\left(F_{(3,29022)}=83.547, p<.001\right)$. Si bien es significativo, es decir, estadísticamente el género, la edad y el nivel de enseñanza están explicando el porcentaje de asistencia, estas variables logran predecir muy poco el modelo para los estudiantes de la modalidad educativa para jóvenes y adultos, tal como se puede apreciar en la tabla 8 .

\section{Tabla 7}

Resumen MRLM sobre asistencia escolar, según género, edad y nivel de enseñanza

\begin{tabular}{cccccccccccc}
\hline & & & & & \multicolumn{6}{c}{ Estadísticos de cambio } \\
\cline { 6 - 11 } Modelo & $R$ & $R^{2}$ & $\mathrm{~B}$ & $\mathrm{E}$ & $\begin{array}{c}\text { Cambio } \\
\text { en } R^{2}\end{array}$ & $\begin{array}{c}\text { Cambio } \\
\text { en } F\end{array}$ & $\mathrm{Gl}_{1}$ & $\mathrm{Gl}_{2}$ & $\begin{array}{c}\text { Sig. Cambio } \\
\text { en } F\end{array}$ & $\begin{array}{c}\text { Durbin- } \\
\text { Watson }\end{array}$ \\
\hline 1 & $.093^{\mathrm{a}}$ & .009 & .008 & 43.07 & .01 & 83.55 & 3 & 29022 & .000 & 1.77 \\
\hline
\end{tabular}

\section{Tabla 8}

Regresión sobre asistencia escolar, según género, edad y nivel de enseñanza

\begin{tabular}{lccccc}
\hline & $\mathrm{B}$ & SE B & $\beta$ & $t$ & $p$ \\
\hline Edad & .30 & .02 & .07 & 12.44 & .00 \\
Nivel de enseñanza & -.02 & .00 & -.04 & -7.13 & .00 \\
Género & 1.81 & .52 & .02 & 3.48 & .00 \\
\hline
\end{tabular}

Ahora, en el segundo MRL propuesto el cual se puede revisar en la tabla 9, compuesto por las variables independientes de Edad, asistencia, género y nivel de enseñanza, encontramos que estas explican un $89 \%$ de la varianza sobre la variable dependiente promedio de notas $\left(F_{(4,29021)}=64532, p<.001\right)$. Los resultados evidenciados en la tabla 10 sugieren que la variable de asistencia $(\beta=.95)$, género $(\beta=.06)$, y nivel de enseñanza $(\beta=.04)$ tienen un peso mayor sobre la dependiente. Lo ante- 
rior, indica que mayoritariamente es la asistencia, seguido por género y nivel de enseñanza los factores que más incide en el promedio general de notas, dejando fuera del modelo a la edad de las personas.

\section{Tabla 9}

Resumen MRLM sobre el promedio de notas según asistencia escolar, género, edad y nivel de enseñanza

\begin{tabular}{lllllllllll}
\hline & & & & \multicolumn{6}{c}{ Estadísticos de cambio } & \\
\cline { 5 - 10 }$R$ & $R^{2}$ & B & E & $\begin{array}{c}\text { Cambio } \\
\text { en } R^{2}\end{array}$ & $\begin{array}{c}\text { Cambio } \\
\text { en } F\end{array}$ & $\mathrm{Gl}_{1}$ & $\mathrm{Gl}_{2}$ & $\begin{array}{c}\text { Sig. Cambio } \\
\text { en } F\end{array}$ & $\begin{array}{c}\text { Durbin- } \\
\text { Watson }\end{array}$ \\
\hline $.95^{\mathrm{a}}$ & .90 & .90 & .83 & .90 & 64532.02 & 4 & 29021 & .00 & 1.45 \\
\hline
\end{tabular}

\section{Tabla 10}

Regresión sobre el promedio de notas según asistencia escolar, género, edad $y$ nivel de enseñanza

\begin{tabular}{lccccc}
\hline & B & SE B & $\beta$ & $t$ & $p$ \\
\hline Porcentaje Asistencia & .06 & .00 & .95 & 506.86 & .00 \\
Edad & .00 & .00 & .00 & .58 & .57 \\
Género & .33 & .01 & .06 & 32.56 & .00 \\
Nivel de enseñanza & .07 & .00 & .04 & 17.88 & .00 \\
\hline
\end{tabular}

\section{Discusión}

A partir de lo expuesto, podemos reconocer que las trayectorias educativas en EPJA son plurales, heterogéneas y complejas en su conjunto. Esto nos habla a su vez de una definición de cómo es entendida la deserción en este recorrido escolar, como una trayectoria diferente a la teórica, a lo que se espera en el desarrollo de estos estudiantes en esta modalidad, donde encontramos un porcentaje no menor de personas, que siguen con rutas diferentes y que no consiguen culminar sus estudios, siendo una opción permanente para esta población la deserción. 
$\mathrm{Al}$ revisar en detalle la caracterización de estas personas y de sus trayectorias escolares en la modalidad para jóvenes y adultos, encontramos en primer lugar que está compuesta principalmente por estudiantes de sexo masculino, entre 15 a 25 ańos, concentrados en la educación secundaria humanista-científica. En cuanto asistencia, hay un tercio de este grupo que asiste menos del $25 \%$ en el año escolar; y en resultados académicos la mayoría obtienen un promedio de notas que está bajo el corte de aprobación. Estos elementos inciden en las tasas finales de aprobación-reprobación, y por tanto impactan en las trayectorias educativas de estos estudiantes, ya que solo la mitad de la población estudiantil analizada, pertenecientes a la modalidad EPJA consiguió avanzar de grado para el ańo 2018.

En el caso de los análisis de relación entre variables, encontramos que existe una correlación alta entre asistencia escolar, promedio de notas, y edad; permitiendo rechazar la hipótesis nula, y confirmando que a mayor edad, mejor asistencia y mayores calificaciones aumenta la tasa de aprobación final, permitiendo que se pueda dar términos a las trayectorias educativas. En cuanto a los modelos de regresión lineal, lo que más está explicando la varianza sobre el promedio de notas y la aprobación del nivel es en primer lugar la asistencia, lo que aumenta la relevancia por abordar la deserción. En segundo lugar, se explica por el género, favoreciendo a las mujeres y por último el nivel de enseñanza, quedando fuera en esta ecuación la edad de las personas. En este contexto, encontramos que efectivamente existen diferencias entre los grupos analizados, identificando plenamente al grupo que está más expuesto a suspender y/o retirarse, por tanto, ver entorpecida nuevamente su trayectoria educativa, estamos hablando de un grupo de riesgo, que mayoritariamente son hombres, que están entre los 19 a 25 años.

La educación para jóvenes y adultos nació bajo un foco de reforma, para asegurar el acceso a educación a una población que se ha visto marginada del sistema educativo y que suele ser muy vulnerable. Se puede observar a partir de los resultados expuestos que los desafíos planteados por la modalidad no están siendo cubiertos, ya que todavía hay un 
amplio margen de personas que no logran los aprendizajes esperados, reprueban su año escolar, e incluso se retiran del sistema. Es necesario transformar esta realidad para avanzar en lo que para algunos autores debiera ser el objetivo principal de las políticas educativas (Terigi, 2014; Echeita, 2008; Blanco, 2006). Esta perspectiva debe superar el marco de la igualdad de oportunidades y políticas compensatorias, orientadas a la recuperación de estudios y capacitación de los empleos tecnificados, debiéndose desarrollar en el marco de la justicia educativa un nuevo modo de desarrollo humano destinado a la creación de las capacidades de las personas y de sus comunidades. Así, estos estudiantes podrán ser protagonistas de sus proyectos propios, podrán expresar y ver reconocida su identidad, experiencia y creatividad propia. Además, también se fomentará el acceso a bienes culturales y tecnológicos, y se desarrollarán en la vida ciudadana, concurriendo a la creación de sociedades más justas mediante un reconocimiento y validación de las diferentes trayectorias educativas existentes (Murillo y Hernández, 2014).

No podemos olvidar que la educación para jóvenes y adultos es un derecho humano y es la puerta al ejercicio de otros derechos. Debe adecuarse al nuevo contexto educacional, entendiendo que recibe a una población cada vez más joven y con necesidades particulares. Las respuestas a problemas cruciales del mundo actual están completamente ligadas a la educación y al aprendizaje permanente de calidad de toda la población siendo EPJA un pilar clave (GIPE, 2018). Para la agenda 2030 de la ONU, la educación es un objetivo primordial, que permitiría incluso facilitar y conseguir lo propuesto, siendo la inclusión y equidad fundamentos para una educación y un aprendizaje de calidad, en pro de garantizar este derecho y promover oportunidades de aprendizaje durante toda la vida para todos (Duk y Murillo, 2018). En este contexto, es que el llamado es a poner atención a este grupo de personas, que son estudiantes que han vuelto a la educación y quieren terminar su trayectoria educativa, ofreciendo las condiciones óptimas y necesarias para sus estudios, que consigan los aprendizajes esperados. Pero también, es necesario entregarles un sentido para que terminen su educación obligatoria, que crean que es posible, debemos recuperar 
la esperanza de la educación, nuestra gestión debe avanzar por esos rumbos y desafíos.

Esta investigación planteó el conocer las trayectorias educativas, reconociendo que los datos solo nos permitían un proxi, estos plantean que es necesario generar una intervención, pero también invitan en su gestión a desarrollar un enfoque preventivo, para reducir los malos resultados y una posible nueva deserción, incorporando modificaciones que motiven a los estudiantes (Trigueros y Navarro, 2019). Esto nos permitiría avanzar, tal como indica Terigi (2014), hacia una visión de proceso y cambio, desde un abordaje crítico que cuestione los procedimientos, sin normalizar el fracaso ni la deserción. Finalmente, quedan pendiente algunos desafíos que apunten a buscar las barreras que están obstaculizando las trayectorias educativas exitosas en la población estudiantil EPJA, identificando aquellos elementos que facilitarían la presencia, participación y aprendizaje (Echeita, 2008) dentro de la modalidad como quiebres que permitan la transformación, mejora y esperanza en la educación.

\section{Referencias}

Ainscow, M. (2017). Haciendo que las escuelas sean más inclusivas. Revista de Educación Inclusiva, 5(1), 39-49.

Aristimuño, A. (2015). El fracaso escolar, ¿fracaso de quién?: la modificación del concepto de fracaso. Revista latinoamericana de educación inclusiva, 9(1), 111-126.

Campero, C. (2018). Contribuciones de la red de educación de personas jóvenes y adultas a la profesionalización de sus integrantes. En DVV (eds.), Papel e impacto de la educación de adultos (pp. 22-28). ICAE.

Carrasco, A. (2013). Mecanismos performativos de la institucionalidad educativa en Chile: pasos hacia un nuevo sujeto cultural. Observatorio Cultural, 15(1), 4-10. 
Castillo, D. Espinoza, O. y González, L. E. (2013). Deserción en la educación primaria: una mirada desde la perspectiva de la equidad y la cohesión social. Politicas públicas para fomentar la equidad y la cohesión social en el sistema educacional: escenarios, dilemas y desafios (pp. 68-88).

Centro de Estudios Mineduc. (2013). Estadísticas de la educación 2012. Santiago: Mineduc. Disponible en https://centroestudios. mineduc.cl/wp-content/uploads/sites/100/2017/07/Estadisticas_de-la-Educacion_2012.pdf

Portales-Olivares, J., Cortés-Rojas, L. y Peters-Obregón, H. (2019). Desescolarización, exclusión educativa y el desafío del reingreso escolar en Chile. Revista Saberes Educativos, (3), 144-153. https://doi.org/10.5354/2452-5014.2019.53792

CPCE. (2016). Informe 4: Estudio niños/as, adolescentes y jóvenes fuera de la escuela. Caracterización y análisis de la demanda para una modalidad de escuelas de segunda oportunidad. Disponible en: http://cpce.udp.cl/wp-content/uploads/2016/07/Informe_ ESO_21032016.pdf

Díaz, O. Campos, J. Guajardo, D. y González, L. (2016). Evaluación de los programas de reescolarización en Chile: la perspectiva de los estudiantes. Educação e Pesquisa, 42(4), 969-986. https://doi. org/10.1590/s1517-9702201605142856

Duk, C. y Murillo, F. (2018). El mensaje de la educación inclusiva es simple, pero su puesta en práctica es compleja. Revista latinoamericana de educación inclusiva, 12(1), 11-13.

Dussaillant, F. (2017). Deserción escolar en Chile. Propuestas para la investigación y la política pública. Análisis-Centro de políticas públicas Facultad de Gobierno UDD, 18.

DVV International. (2018). Papel e impacto de la educación de adultos. ICAE.

Echeita, G. y Verdugo, M. (2004). La Declaración de Salamanca sobre necesidades educativas especiales 10 años después. Valoración y prospectiva. INICO. 
Echeita, G. (2008). Inclusión y exclusión educativa:"voz y quebranto”. REICE. Revista Electrónica Iberoamericana sobre Calidad, Eficacia y Cambio en Educación, 6(2), 9-18.

Espinoza, O. Castillo, D. González, L. y Santa Cruz, J. (2014). Educación de adultos e inclusión social en Chile. Psicoperspectivas, 13(3), 69-81.

Espinoza, O. González, L. Castillo, D. y Neut, S. (2019). Condicionantes de la retención estudiantil en «escuelas de segunda oportunidad" en Chile. Revista Latinoamericana de Ciencias Sociales, Niñez y Juventud, 17(2), 1-27.

Fardella, C. (2013). Resistencias cotidianas en torno a la institucionalización del modelo neoliberal en las políticas educacionales: El caso de la docencia en Chile. Psicoperspectivas, 12(2), 83-92.

GIPE. (2018). La sociedad civil por la promoción y defensa del derecho a la educación con las personas jóvenes y adultas. Propuesta de agenda a las coaliciones politicas y candidatos. GIPE.

Guerrero, A. (16 de diciembre de 2017). Educación para adultos: la cara olvidada y precarizada. La Izquierda Diario. Recuperado de https://www.laizquierdadiario.cl/ Educacion-para-adultos-la-cara-olvidada-y-precarizada

Infante, M. I. y Letelier, M. E. (2013). Alfabetización y educación: lecciones desde la práctica innovadora en América Latina y el Caribe.

Mayer, L. y Cerezo, L. (2016). Tutorías y estipendio mensual: contribuciones a la trayectoria universitaria de jóvenes en situación de vulnerabilidad. Revista Latinoamericana de Ciencias Sociales, Niñez y Juventud, 14(2), 1421-1433.

Mineduc. (2016). Subvención escolar preferencial. Recuperado de: https://sep.mineduc.cl/

Mineduc. (2017). Educación de personas jóvenes y adultas. Recuperado de https://epja.mineduc.cl/

Mineduc. (2018). Bases de datos oficiales. Recuperado de: https:// centroestudios.mineduc.cl/2018/11/28/ bases-de-datos-oficiales-2018-disponibles/ 
Muñoz, S. y Acuña,V. Caracterización de los jóvenes usuarios de la educación de adultos en la Región de Valparaíso, Chile. Sociedad Hoy, 24, 33-47.

Murillo, F. y Hernández, R. (2014). Liderando escuelas justas para la justicia social. Revista Internacional de Educación para la Justicia Social (RIEJS).

OCDE (2015). Education Policy Outlook 2015: Making Reforms Happen. París: OECD Publishing.

OCDE (2016). PISA 2015, resultados clave. Recuperado de https:// www.oecd.org/pisa/pisa-2015- results-in-focus-ESP.pdf

ONU (2015a). Asamblea general, Objetivos de desarrollo Sostenible. Recuperado de: https://www.un.org/sustainabledevelopment/ es/education/

ONU (2015b). Educación para todos. Recuperado de: https:// www.un.org/sustainabledevelopment/es/events/ foro-mundial-sobre-la-educacion-2015/

Opazo, A. (2017). Trayectoria escolar de los estudiantes más vulnerables del sistema educativo chileno. Santiago: Centro de Estudios Mineduc.

Soto, R. Mera, J. Nuñez, C. Sisto, V. y Fardella, C. (2016). Entre la efectividad y los afectos: nuevos docentes en tiempos de nuevo management público. Athenea Digital, 16(3), 3-19.

Terigi, F. (2007). Los desafíos que plantean las trayectorias escolares. Paper presentado en el III Foro Latinoamericano de educación. Jóvenes y docentes. La escuela secundaria en el mundo de hoy. Fundación Santillana. Recuperado de: http://www.bibliopsi. org/docs/carreras/obligatorias/CFP/educacional/erausquin/ Unidad\%204/Educacional\%20Erausquin \%20-\%20\%20 Terigi\%20-\%20Los\%20desafios\%20que\%20 plantean $\% 20$ las\%20trayectorias\%20escolares.pdf

Terigi, F. (2012). VIII Foro Latinoamericano de Educación: saberes docentes: qué debe saber un docente y por qué. Fundación Santillana. Recuperado de https://www.fundacionsantillana.com/ PDFs/8vo_foro.pdf 
Terigi F. (2014). Trayectorias escolares e inclusión educativa: del enfoque individual al desafío para las políticas educativas. En A. Marchesi, R. Blanco y L. Hernández (Coords.), Avances y desafios de la educación inclusiva en Iberoamérica (pp. 71-90). OEI.

Trigueros, R. y Navarro, N. (2019). La influencia del docente sobre la motivación, las estrategias de aprendizaje, pensamiento crítico y rendimiento académico de los estudiantes de secundaria en el área de Educación Física. Psychology, Society, \& Education, 11(1), 137-150.

Unesco (2015). Recomendación sobre el aprendizaje y la educación de adultos. Recuperado de http://portal.unesco.org/es/ev.phpURL_ ID $=49354 \&$ URL_DO=DO_TOPIC\&URL_SECTION=201. html

Unesco (2017). El poder del aprendizaje y la educación de adultos en todo el mundo. Revisión de la sexta Conferencia Internacional de Educación de Adultos. Recuperado de http://www.unesco.org/ new/es/santiago/press-room/single-new/news/el_poder_del_ aprendizaje_y_la_educacion_de_adultos_en_todo/

Unesco (2019). La ONU recibe al "Amigo Invisible: Educación de Adultos y los Objetivos de Desarrollo Sostenible» en Nueva York. Recuperado de https:/uil.unesco.org/es/educacion-adultos/ onu-recibe-al-amigo-invisible-educacion-adultos-y-los-objetivos-desarrollo

Recibido: 2020-03-05

Revisado: 2021-07-01

Aceptado: 2021-07-12 